\title{
An Outside-Mouth Measurement System for the Gap Detect of Dental Prosthesis Elements
}

\author{
Shinn-Liang Chang*, Duy-Hoang Nguyen, Bing-Jiun Tsai \\ Department of Power Mechanical Engineering, National Formosa University, Huwei, Yunlin, Taiwan. \\ * Corresponding author. Tel.: +886-5-6315440; email: changsl@nfu.edu.tw. \\ Manuscript submitted December 29, 2016; accepted March 10, 2017. \\ doi: 10.17706/ijbbb.2017.7.2.125-132
}

\begin{abstract}
The possible ways to measure the gap between dental prosthesis element and human tooth are measured by contact of probe or image processing. However, the gap measuring instrument to detect the denture installation is still in development. Nowadays, in order to judge whether the gap of denture is too large or not, dentists use a needle to scrape on the surface of denture. This way is easily affected by the applied force and causes misjudgment. Therefore, this study aims to develop a device to detect the gap of dental, such the device is not found currently in industry.

Based on the requirement, this study applies image processing to develop an Outside-Mouth Measurement System (OMMS) for gap measurement of dental prosthesis element outside the mouth. The system functions use image filtering, image morphology, and edge detection to capture and calculate the contour for the gap measurement of dental. The developed measurement system could combine with the oral camera to improve its application. The results in this study are efficient to measure the gap of dental prosthesis elements.
\end{abstract}

Key words: Dental, tooth, image processing, measurement system.

\section{Introduction}

Nowadays, with the rapid development of industrial technology and social economy, people care about their dental health much more than ever. This make the dentists hoping to pursue a more accurate gap detection technology in measuring the gap between two elements. Laser scanning technology is adopted in measurement of coordinate and surface reconstruction [1], [2]. However, the shadowing and reflection of certain material quality and surface treatment cause the difficulty of measurement. Huertas [3] presented a system that takes a grey level image as input, located edges with sub-pixel accuracy, and linked them into lines. A finding zero-crossings method was used to obtain the edges from the full resolution image. Finally, these edges are grouped into lines by using linear technique. Pentland [4] proposed a novel method of obtaining a depth of field that is simultaneously fast enough, reliable enough, and produces a sufficiently dense depth map to support the requirements of a robot moving in a complex environment. Nayar and Nakagawa [5] presented two algorithms for depth estimation. The first algorithm simply looks for the focus level that maximizes the focus measured at each image point. The second algorithm uses a Gaussian model to interpolate the focus measured to obtain more accurate depth estimates. They used the sum-modified-Laplacian (SML) operator which is developed to compute local measurement of image focus. The image sequence and the focus measurement obtained at each image point are used to compute local depth estimates. Subbarao and Choi [6] proposed a new concept named Focused Image Surface (FIS). FIS of 
an object is defined as the surface formed by the set of points at which the object points are focused by a camera lens. The new method has been implemented on an actual camera system, and the results of shape recovery and focused image reconstruction are presented. Shrivakshan and Chandrasekar [7] studied with some edge detection techniques by using MATLAB software. The case study deals with observation of Shark Fish Classification through image processing using the various filter. Soft Computing is an emerging field that consists of complementary elements of fuzzy logic, neural computing and evolutionary computation. Based on the fuzzy logic, genetic algorithm and neural network, Senthilkumaran and Rajesh [8] proposed the theory survey of edge detection for image segmentation. Gao, et al. [9] proposed a method which combines Sobel edge detection operator and soft-threshold wavelet de-noising to do edge detection on images including White Gaussian noises. Recently, dentists notice the manufacture of dental prosthesis elements by 3D printing. However, the shrinkage is the main problem in 3D printing [10]. This improves the requirement of the developing of gap detect devices. In summary, edge detection is the main objective considering the changes of grey image. Image edge is the most basic features of the image processing technique.

In previous study [11], a detection device and computation algorithm were developed successfully to detect the edge of dentures gap. This study aims to develop a software of OMMS with User Interface (UI) and also upgrade the camera device to detect the gap of dental by applying image processing techniques and numerical methods. The results obtained may open up a new direction for dental science.

\section{Image Measurement System}

The developed system in this research applies the image processing techniques and numerical methods for the detection of dental prostheses gap outside the human mouth. The measurement system contains a single CCD (Charge-coupled Device) to capture the gap of dentures, and the denture image data is transferred to the computer through the video capture card. Then, the image processing techniques and numerical methods are applied to calculate the gap size of the dentures. The system structure flowchart is shown in Fig. 1 [10].

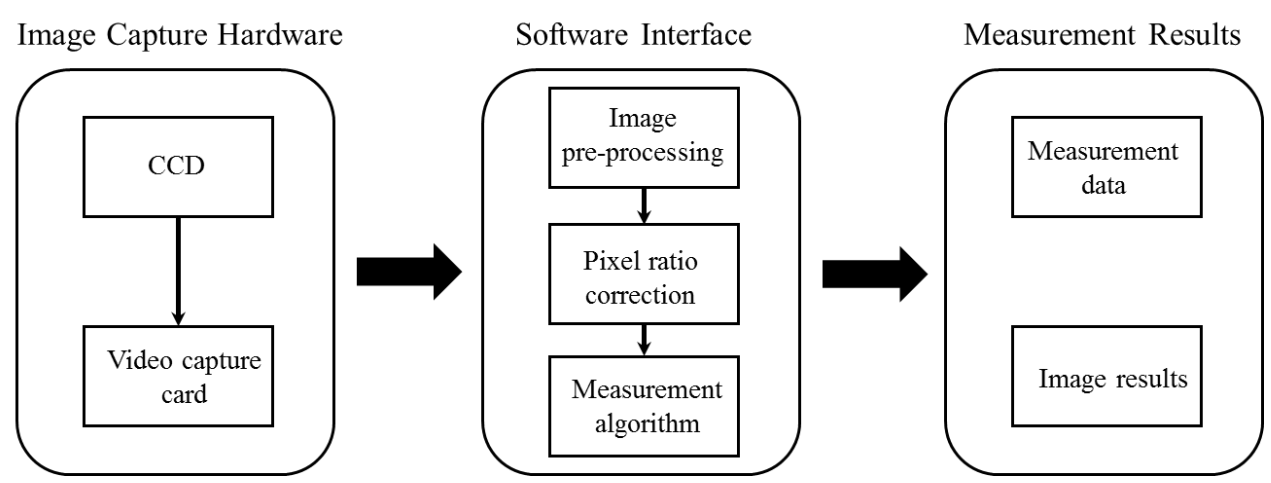

Fig. 1. Structure of machine vision system.

The developed program shown in Fig. 2 and Fig. 3 is based on the application of Microsoft Visual Studio 2015. The software features are mainly contained read image, image processing, computed geometry characteristics, and the output of measurement results. As shown in Fig. 2 and Fig. 3, the image of teeth was imported into the software firstly. Then after image processing, the image will be computed for its geometry to obtain the gap measurement that shown in Fig. 3.

The developed measurement software got the digital images of dentures gap from the video capture card and then loaded into the measurement program to detect the gap size. The boundary contours are obtained 
to facilitate the calculation of dentures gap and shown in Fig. 4. The image processing steps are shown in Fig. 5 [10].

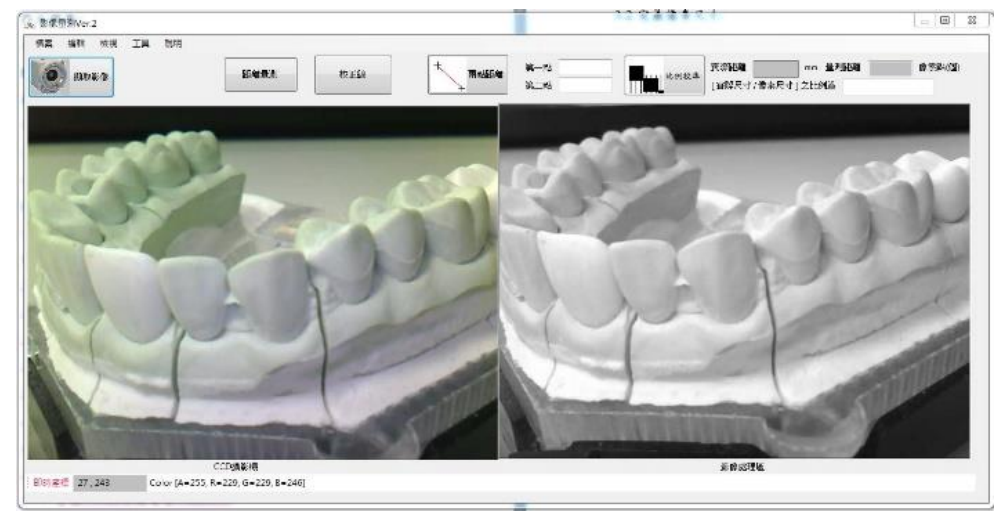

Fig. 2. Measurement system interface.

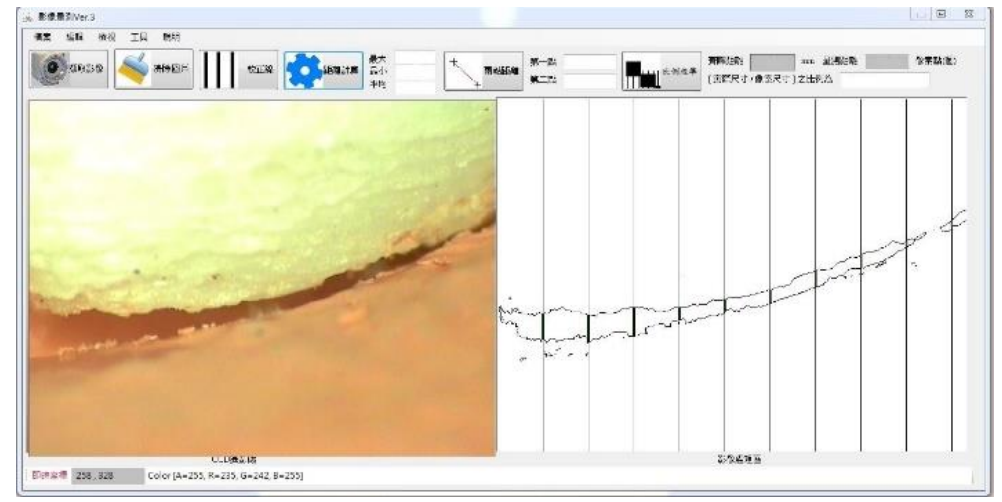

Fig. 3. Gap measurement in the interface.
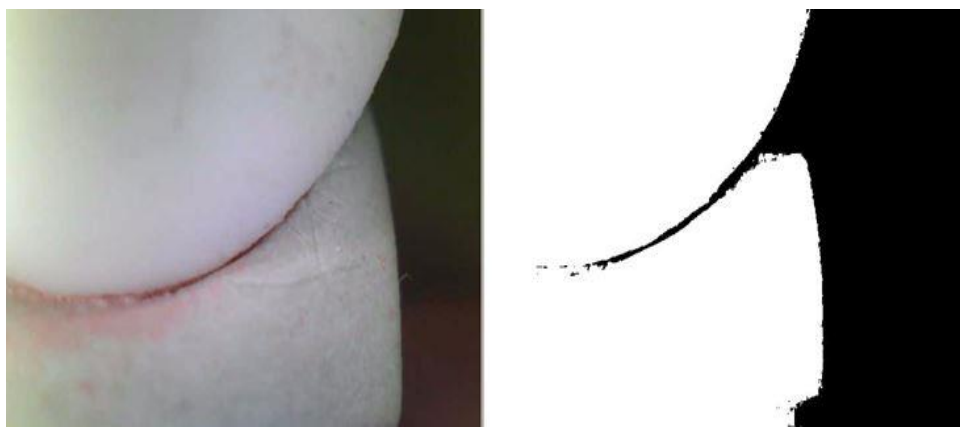

Fig. 4. Boundary contour of tooth.

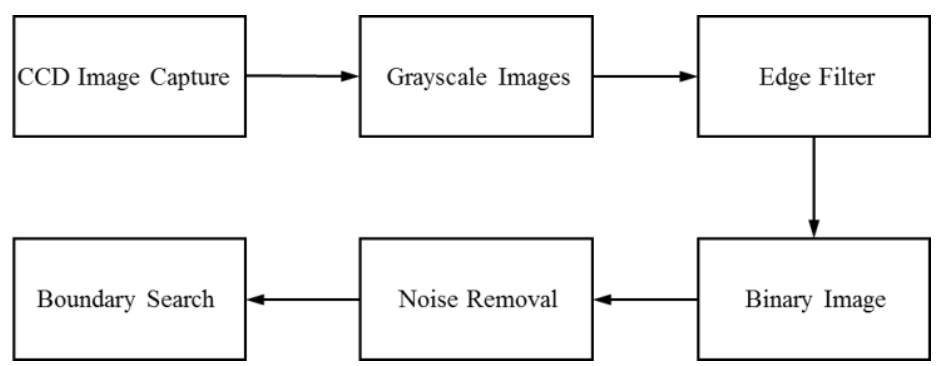

Fig. 5. Flow chart of the image processing.

In order to measure the denture gap, following the image processing step shown in Fig. 5, the image of 
gap captured by CCD will be transformed into grayscale and placed into coordinate of measurement scale system, as shown in Fig. 6. By boundary search method, the software then record the intersection points between the contour of the gap and the baseline. The intersection points are the red points located on the boundary of the gap as shown in Fig. 7. The distance between every upper and lower points then were used to compute the size of the gap (Fig. 7).

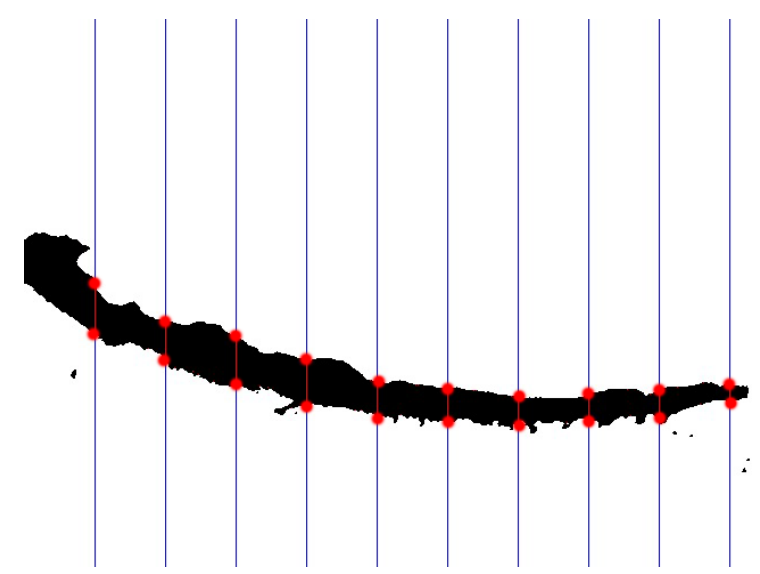

Fig. 6. Baseline used for measuring the gap.

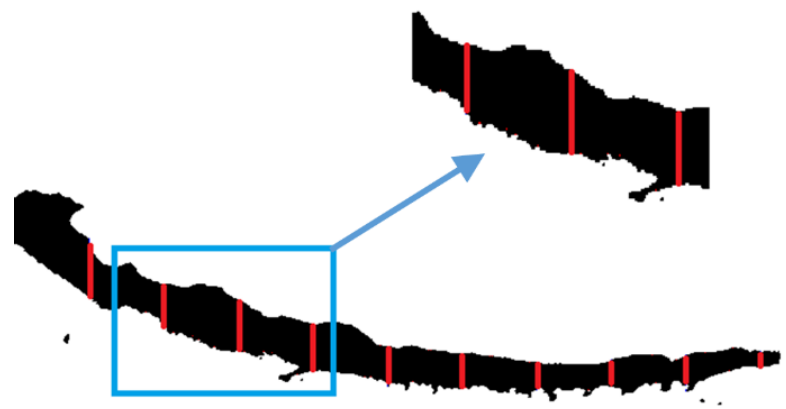

Fig. 7. The gap after image processing step.

\section{Pixel Correction}

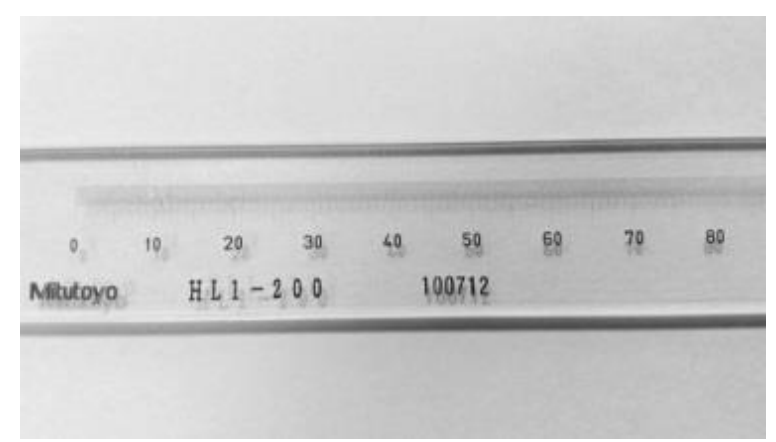

Fig. 8. Local image of glass calibration scale.

In the image, the pixel coordinate represents the position of each pixel. The algorithms are developed to calculate the distance of pixels and then converted into the actual size of dentures gap. Here the pixel arithmetic is adopted to calculate the dentures gap size and error. In order to transfer the distance of digital image into the corresponding distance, the ratio between the pixel distance and actual size must be 
determined. $\mathrm{P}_{\mathrm{m}}$ shown in the following equation is defined as the scale.

$$
P_{m}=\frac{\text { actual distance }}{\text { pixel distance }}
$$

In calibration experiment, a Mitutoyo $200 \mathrm{~mm}$ standard measuring scale shown in Fig. 8 was used to correct the resolution of pixel. As shown in Fig. 9, the distance between A and B in the image represents 1 $\mathrm{mm}$. The ratio between the pixel distance and actual size can be easily calculated by equation (1).

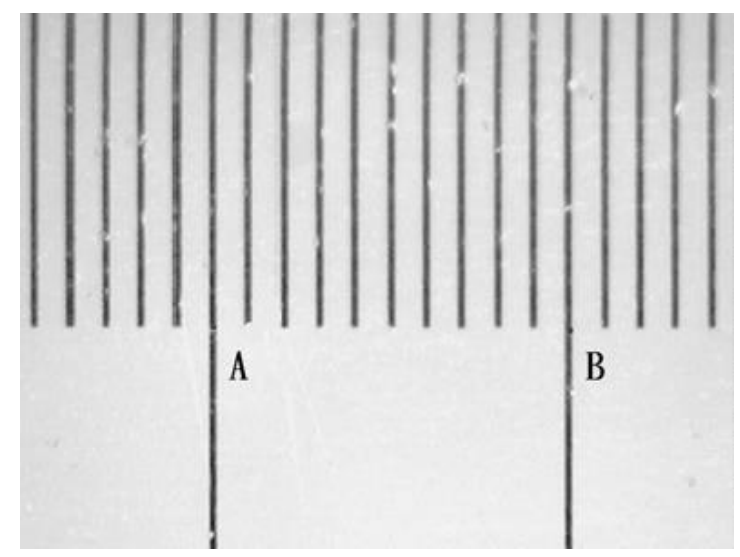

Fig. 9. Pixel resolution correction mode.

\section{Experiment Measurement and Results}

The measurement system - OMMS developed in this study can be applied in the gap measurement of dental prosthesis element for the outside mouth measurement instrument, oral measurement, and the 2.5D image measurement instrument shown in Fig. 10 and Table I, to verify the reliability of the system.

Some experiments measurement were taken to verify the ability of the OMMS. In the experiments, the tooth model printed by the 3D printer was used as the target of the measurement test, as shown in Fig. 11a.

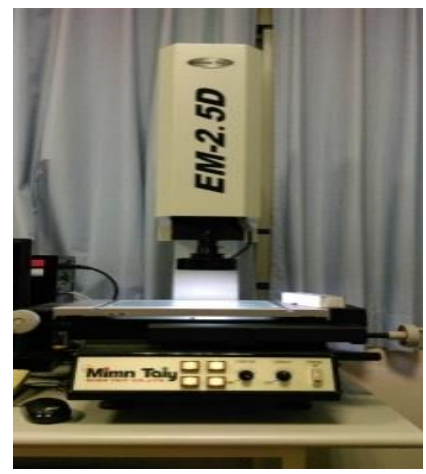

Fig. 10. 2.5D image measurement instrument.

The measurements have three different lenses to capture gap image on the same dental model. The gap image is taken at ten positions from left side to right side of prosthesis element shown in Fig. 11b. The largest gap will affect the quality of dentures. Therefore, the measurements are focused on the maximum gap. Due to the differences in focal length of the lens, the measurements have different pixel scales. The pixel scale of oral measurement instrument is $0.007462(\mathrm{~mm} / \mathrm{pixel})$, the outside mouth measurement instrument is 0.003205 (mm/pixel), and 2.5D image measurement instrument is 0.003450 (mm/pixel). 
Table 1. Lens Specifications

\begin{tabular}{cc}
\hline \hline Resolution & $0.0005 \mathrm{~mm}$ \\
\hline CCD & Color $1 / 3 \mathrm{CCD}$ \\
\hline Magnification & $4.5 \mathrm{X}$ \\
\hline \hline
\end{tabular}

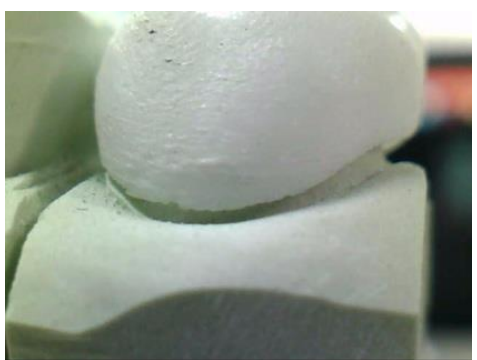

(a) Model Printed by 3D Printer

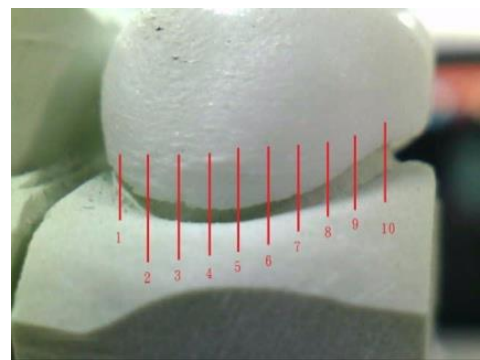

(b) Diagram of Measurement Position.

Fig. 11. The tooth model.

Table 2. Measurement Results of Oral Measurement Instrument

\begin{tabular}{cccc}
\hline & Pixel Scale & Maximum Gap & $\begin{array}{c}\text { The gap } \\
\text { average }\end{array}$ \\
\hline 1 & & 0.223870 & 0.134320 \\
2 & & 0.238784 & 0.145870 \\
3 & & 0.243580 & 0.148190 \\
4 & & 0.246246 & 0.149240 \\
5 & 0.007462 & 0.246246 & 0.150090 \\
6 & $(\mathrm{~mm} / \mathrm{pixel})$ & 0.253708 & 0.151640 \\
7 & & 0.253730 & 0.154212 \\
8 & & 0.253730 & 0.156702 \\
9 & & 0.261170 & 0.156702 \\
10 & & 0.276094 & 0.158701 \\
\hline \hline
\end{tabular}

Table 3. Measurement Results of the Outside Mouth Measurement Instrument

\begin{tabular}{cccc}
\hline$\searrow$ & Pixel Scale & Maximum Gap & $\begin{array}{c}\text { The gap } \\
\text { average }\end{array}$ \\
\hline 1 & & 0.126854 & 0.089544 \\
2 & & 0.134316 & 0.097006 \\
3 & & 0.137506 & 0.104418 \\
4 & & 0.140631 & 0.104464 \\
5 & 0.003205 & 0.141778 & 0.110416 \\
6 & $(\mathrm{~mm} /$ pixel) & 0.142857 & 0.112392 \\
7 & & 0.149240 & 0.119392 \\
8 & & 0.164164 & 0.120104 \\
9 & & 0.173708 & 0.126854 \\
10 & & 0.179088 & 0.129392 \\
\hline \hline
\end{tabular}

Table 4. Measurement Results of 2.5D Image Measurement Instrument

\begin{tabular}{cccc}
\hline$\backslash$ & Pixel Scale & Maximum Gap & $\begin{array}{c}\text { The gap } \\
\text { average }\end{array}$ \\
\hline 1 & & 0.141034 & 0.093103 \\
2 & & 0.151724 & 0.098214 \\
3 & & 0.158620 & 0.108027 \\
4 & & 0.158620 & 0.113071 \\
5 & 0.003450 & 0.165517 & 0.118755 \\
6 & (mm/ pixel) & 0.165517 & 0.122457 \\
7 & & 0.172548 & 0.125028 \\
8 & & 0.179421 & 0.126698 \\
9 & & 0.193214 & 0.129870 \\
10 & & 0.196664 & 0.131320 \\
\hline \hline
\end{tabular}




\section{Conclusion}

In Table 2-Table 4, the instrument for the measurement of outside the mouth has higher resolution and magnification in lens. It can capture the gap image with higher revolution than oral measurement instrument and 2.5D image measurement instrument. A smaller pixel will cause the measurement with higher accuracy.

The differences of measurement got from the oral measurement instrument and the outside mouth measurement instrument are about $0.05 \mathrm{~mm}$. The error of maximum gap among three kinds of gap measurement is about $0.1 \mathrm{~mm}$. The measurement accuracy of oral measurement instrument is lowest due to the lowest resolution of the lens.

\section{Acknowledgment}

The authors wish to thank MOST 105-2218-E-010-002 project for the financial support.

\section{References}

[1] Manthey, D. W., Knapp, K. N., \& Lee, D. (October 1994). Calibration of a laser range finding coordinate measuring machine. Opt. Eng., 33(10), 3272 -2379.

[2] Bradley, C., \& Vickers, G. W. (September 1993). Free-from surface Reconstruction for machine vision rapid prototyping. Opt. Eng., 32(9), 2191-2200.

[3] Huertas, A. (September 1986). Detection of intensity changes with sub-pixel accuracy using Laplacian-Gaussian masks. IEEE Trans. on Pattern Analysis and Machine Intelligence, 8(5), 651-664.

[4] Pentland, A. P. (July 1987). A new sense for depth of field. IEEE Transactions on Pattern Analysis and Machine Intelligence, 9(4), 523-531.

[5] Nayar, S. K., \& Nakagawa, Y. (1990). Shape from focus: An effective approach for rough surfaces. IEEE International Conference on Robotics and Automation, 2(10), 218-225.

[6] Subbarao, M., \& Choi, T. (March 1995). Accurate recovery of three-dimensional shape from image focus. IEEE Transaction on Pattern Analysis and Machine Intelligence, 17(3), 266-274.

[7] Shrivakshan, G., \& Chandrasekar, C. (September 2012). A comparison of various edge detection techniques used in image processing. International Journal of Computer Science Issues, 9(5), 269-276.

[8] Senthilkumaran, N., \& Rajesh, R. (May 2009). Edge detection techniques for image segmentation and a survey of soft computing approaches. International Journal of Recent Trends in Engineering, 1(2), 250-254.

[9] Gao, W. S., Yang, L., Zhang, X. G., \& Liu, H. Z. (July 2010). 2010 3rd IEEE International Conference on Computer Science and Information Technology, Vol. 5, (pp. 9-11, 67-71).

[10] Hsu, H. J., Lee, S. Y., et al. (December 2016). Shrinkage prediction using finite element analysis and experimental validation using three-dimension slurry printing system. The International Journal of Advanced Manufacturing Technology, 87, 1-8.

[11] Chang, S. L., et al. (April 2016). The image measurement to indicate the gap of dental prosthesis element. Proceedings of 2016 International Conference on Advances in Software, Control and Mechanical Engineering (pp. 12-13, 35-40).

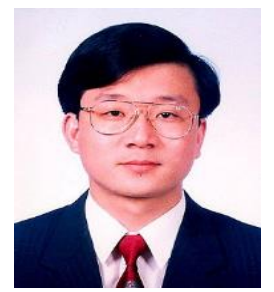

Shinn-Liang Chang is distinguished professor. Chang graduated from National Cheng Kung University for M.E. in 1987 and received his master degree from the Department of Power Mechanical Engineering, National Tsing-Hua University in 1992. Then, he obtained his Ph.D. in mechanical engineering from National Chiao Tung University in 1996.

He had worked as vice manager of R\&D Department and Customer Service Department 
in Luren Precision Co. located in Hsin-Chu Science Based Industrial Park in Taiwan from 1996 to 1998. Since 2005 to 2013, he experienced as the chair of the Department of Power Mechanical Engineering, chair of Center of Design and Application of MEMS, chair of the Institute of Mechanical and Electro-Mechanical Engineering, Dean of Academic Affairs, and Vice President of National Formosa University. He is currently a distinguished professor of Power Mechanical Engineering at National Formosa University in TAIWAN. His research interests include theory of gearing and its application, machine tool design and manufacture, mechanism design and analysis, CAE, 3-D printing, remote inspection and diagnosis. Until now, he publishes over than one hundred technical papers.

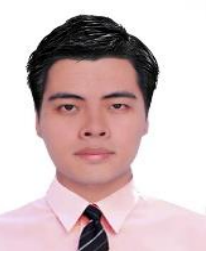

Duy-Hoang Nguyen was born on June 2, 1988, in Hanoi, Vietnam. He graduated from Ho Chi Minh city University of Technology for the bachelor degree in the Department of Apply Science. He received his M.S from the Department of Automation Engineering in Da-Yeh University, Taiwan in 2013. From 2013 to now, he is a Ph.D. student in National Formosa University and do research under the direction of professor Shinn-Liang Chang.

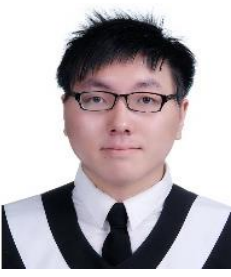

Bing-Jiun Tsai was born in Taipei, Taiwan. He graduated from Oriental Institute of Technology for the bachelor degree in the Department of Mechanical Engineering in 2014. From 2014 to 2016, he is a master student in National Formosa University. He graduated and received his M.S. in the Department of Power Mechanical Engineering in September, 2016. 